Research Article

\title{
The Role of Endothelial Mesenchymal Transformation on Infantile Hemangioma
}

\author{
Xin Zhong, ${ }^{1,2}$ Gang Zheng, ${ }^{2}$ Min Ding, ${ }^{2}$ Jing-Bing Li, ${ }^{2}$ and Qi-Hong Qian (D) ${ }^{1}$ \\ ${ }^{1}$ Department of Dermatology, The First Affiliated Hospital of Soochow University, Suzhou, Jiangsu 215006, China \\ ${ }^{2}$ Department of Dermatology, XuZhou Central Hospital, XuZhou, Jiangsu 221009, China \\ Correspondence should be addressed to Qi-Hong Qian; chhl971@163.com
}

Received 15 February 2021; Revised 16 March 2021; Accepted 17 April 2021; Published 29 April 2021

Academic Editor: Qinhu Zhang

Copyright (c) 2021 Xin Zhong et al. This is an open access article distributed under the Creative Commons Attribution License, which permits unrestricted use, distribution, and reproduction in any medium, provided the original work is properly cited.

Objective. Infantile hemangioma (IH) is the most common infantile benign tumor derived from endothelial cells (ECs). The mechanism of IH degeneration into fibroadipose tissue is still unclear. We aimed to explore the effects of endothelial mesenchymal transformation (EndMT) on IH and explore its underlying mechanism. Methods. HemECs were treated with different concentrations of transforming growth factor- $\beta 1$ (TGF- $\beta 1$ ) as in vitro models. The CCK- 8 assay was utilized to explore the effect of TGF- $\beta 1$ on cell proliferation. Scratch test was conducted to determine the role of TGF- $\beta 1$ in cell invasion. The influence of TGF- $\beta 1$ on the E-cadherin, vimentin, N-cadherin, and LC3B expression in HemECs was detected by Western blot analysis. Expression of TGF- $\beta 1$, E-cadherin, vimentin, N-cadherin, and LC3B in IH was determined by immunohistochemistry. Results. After TGF- $\beta 1$ treatment, HemECs lost pebble-like morphology and presented fusiform or fibroblast-like appearance; the proliferation activity decreased significantly, endothelial cell markers decreased; interstitial markers increased, and autophagy protein expression increased. Immunohistochemical staining demonstrated higher positive expression rate of TGF- $\beta 1$ and N-cadherin and poorer positive expression rate of E-cadherin related to proliferative tissues. In addition, compared with proliferative cells, the positive expression rate of E-cadherin in degenerated tissues was significantly increased, while the positive expression rate of $\mathrm{N}$-cadherin was markedly reduced. Interestingly, the immunohistochemical results indicated that LC3B expression in the proliferative phase was lower than that in the regressive phase, whereas the expression of LC3B was significantly upregulated after hemangioma endothelial cells transformed into endothelial mesenchyme. Conclusion. TGF- $\beta 1$ could induce EndMT in HemECs. TGF- $\beta 1$ inhibited cell proliferation in a concentration-dependent manner and promoted autophagy and migration. Our investigation demonstrated that EndMT plays a significant role in the degradation of hemangioma, which provides an insight for the treatment of hemangioma.

\section{Introduction}

Infantile hemangioma (IH), also known as "strawberry hemangioma," is one of the most common benign endothelial cell-derived tumors in infancy, with incidence about $5-10 \%$ [1]. The main pathological features of IH are hyperplastic endothelial cells and abnormal formation of microvessels. The tumor grows rapidly in the proliferative stage, which can compress the surrounding tissues and organs, cause blindness and dyspnea, and even threaten life. During the course of rapid proliferation, the tumor body formed more microvessels and then entered the stage of regression. Meanwhile, the fibroadipose tissue increased and replaced the hemangioma cell mass [2]. However, the specific mechanism of hemangioma degeneration is still absolutely unclear.

Endoplasmic transformation (ENDMT) refers to the transformation of endothelial cells into mesenchymal cells through a series of molecular changes, along with the inhibition of epithelial cell markers (such as E-cadherin and $\mathrm{CDH} 1$ ), and activation of stromal markers including vimentin, $\alpha$-smooth muscle actin ( $\alpha$-SMA), $\mathrm{N}$-cadherin, and fibroblast specific protein 1 . This physiological and pathological change is closely related to remodeling of vascular microenvironment [3-5], atrioventricular valve development [6], angiogenesis [7], and cardiac fibrosis [8]. Relevant 
efforts have indicated that the level of transforming growth factor- $\beta 1$ (TGF- $\beta 1$ ) is downregulated in proliferative phase, while upregulated in regression phase and could induce the expression of $\alpha$-SMA in endothelial cells [9]. Meanwhile, it has been reported that M1 polarized macrophages can induce EndMT and promote the regression of hemangioma. Moreover, macrophage cytokine (such as TNF- $\alpha$ or IL- $1 \beta$ or IFN- $\gamma$ ) could also induce EndMT and promote the hemangioma involution [10].

These evidences suggest that EndMT has implications for the regression of hemangioma. Nevertheless, as the main inducer of EndMT, the relationship between TGF- $\beta 1$ and hemangioma has not been fully elucidated.

In this work, we isolated and cultured the hemangioma endothelial cells from the proliferated infant blood vessels and induced the EndMT of hemangioma endothelial cells by TGF- $\beta 1$. Also, we further verified it in hemangioma tissue samples. These efforts will provide a theoretical basis and a new insight for TGF- $\beta 1$ to induce endothelial cells to transform into the stroma and promote the regression of hemangioma.

\section{Results}

2.1. TGF- $\beta 1$ Induces EndMT in HemECs. We established a reproducible in vitro EndMT model in HemECs using TGF$\beta 1$ [11]. Microscopically, the HemECs in the blank control group were pebble-like, with irregular polygon, clear boundary, and close connection between cells; after TGF- $\beta 1$ group cells are cultured, the cell morphology has changed significantly, with elongated cells showing fusiform change, fuzzy boundary, and large gap. The results showed that there were quantifiable morphological marker changes in HemECs. Endothelial cells lost pebble-like morphology and presented fusiform or fibroblast-like appearance (Figure 1).

2.2. TGF- $\beta 1$ Inhibits the Proliferation of HemECs. To detect the influence of TGF- $\beta 1$ on HemECs proliferation, different concentrations of TGF- $\beta 1$ administration were utilized in HemECs. The CCK- 8 results showed that TGF- $\beta 1(0.5,5,10$, and $20 \mathrm{ng} / \mathrm{ml}$ ) had significant difference in the proliferation of HemECs (F2 H = 0.68, $P>0.05 ; \mathrm{F} 12 \mathrm{~h}=33.69, P<0.05 ; \mathrm{F}$ $24 \mathrm{~h}=69.35, P<0.05)$. As shown in Figure 2, the proliferation activity of TGF- $\beta 1$ cells in the low dose group was markedly higher as compared with that in the blank control group, while the proliferation rate of TGF- $\beta 1$ cells in the medium and high dose group $(5,10$, and $20 \mathrm{ng} / \mathrm{ml})$ was significantly decreased than that in the blank control group in a concentration-dependent fashion $(P<0.05)$.

2.3. TGF- $\beta 1$ Promotes the Migration of HemECs. The results of the scratch test showed that the scratch distance of cells significantly decreased after stimulated with $5 \mathrm{ng} / \mathrm{ml}$ TGF- $\beta 1$ for 24 hours, compared with the blank control group $(P<0.001)$ (Figure 3).
2.4. The Effect of EndMT on the Expression of Endothelial and Mesenchymal Cell Markers. Previous reports showed that the epithelial cell markers (such as E-cadherin) were inhibited and mesenchymal markers were activated during EndMT (Kovacic et al., 2019; Liang et al., 2016). We performed Western blot to determine related protein expression in EndMT induced by TGF- $\beta 1$. As shown in Figure 4(a), E-cadherin and $\mathrm{N}$-cadherin expression were markedly decreased, while the expression of vimentin was obviously increased in EndMT than that in the control group. In addition, we also detected the expression of related autophagy proteins. The results showed that LC3 and LC3-II/ LC3-I protein expression increased significantly after administration of TGF- $\beta 1$. IHC analysis showed LC3 expression was significantly higher in regressive tissues (Figures 4(b) and 4(c)).

2.5. Structural Changes of Hemangioma Tissue during EndMT. The results of HE staining indicated that hemangioma cells in the proliferative stage were irregular in shape, bulky in volume, clear in boundary, and more microvessels in cells, less interstitial tissue in cell clusters, and microvessels with different diameters could be seen. The cells were arranged in disorder and dense, with large nuclei and deep staining. In contrast, there were more mesenchymal tissues between the hemangioma cell clusters in the regression period. The boundary between EC cluster and fatty tissue is not obvious, loose, and disordered. Some endothelial cells form dense microvascular clusters, blood sinuses are irregular, some endothelial cells form honeycomb, and fibrous adipose tissue increases (Figure 5).

2.6. Expression of Related Genes in Hemangioma during EndMT. To further explore the potential molecular mechanism of $\mathrm{IH}$ activating EndMT, we examined the protein expression of TGF- $\beta 1$, E-cadherin, and N-cadherin in $\mathrm{IH}$ tissues. The immunohistochemistry staining showed that the positive expression rate of TGF- $\beta 1$ was considerably higher in $\mathrm{IH}$ tissues relative to proliferative tissues (see Figure 6; Table $\left.1 ; \chi^{2}=7.342, P<0.01\right)$. In addition, we also analyzed the expression of E-cadherin and $\mathrm{N}$-cadherin protein in 43 cases of hemangioma. Compared with proliferative tissues, the positive rate of E-cadherin was markedly decreased $\left(\chi^{2}=17.922, P<0.05\right)$, while the positive rate of $\mathrm{N}$-cadherin was significantly increased in the regression period $\left(\chi^{2}=24.448, P<0.01\right)$ (Table 2$)$. Consistent with the above results, we obtained the same results in vitro experiments.

We further explored the association between the expression of E-cadherin and $\mathrm{N}$-cadherin and the clinicopathology in IH. We found that the protein levels of E-cadherin and $\mathrm{N}$-cadherin in hemangioma were not significantly different from those in sex, size, depth, and type of hemangioma but significantly different from those in stage and age (Table 2, $P<0.01$ ). In addition, an inverse correlation was found with E-cadherin level and $\mathrm{N}$-cadherin level (rs $=-0.886, P<0.01$ ). 

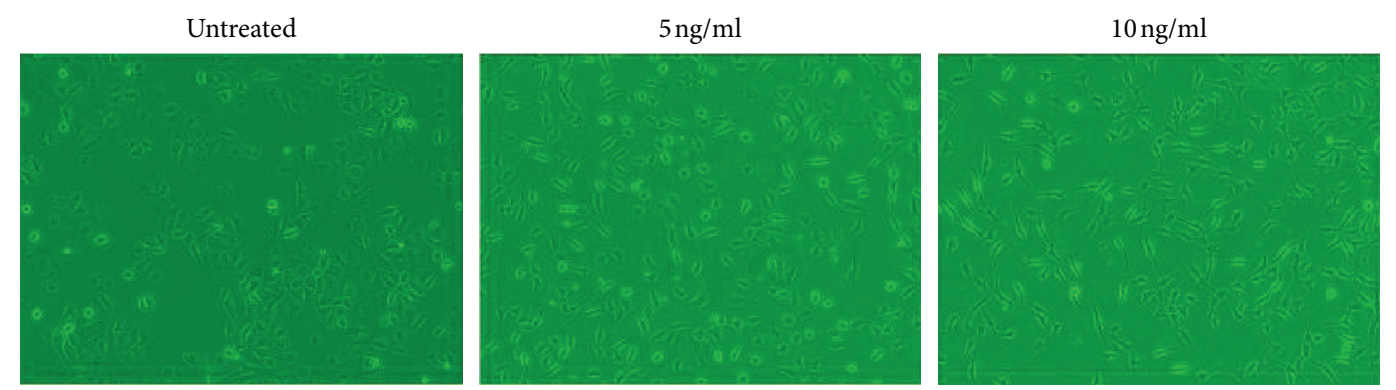

FIgURE 1: TGF- $\beta 1$ induces EndMT in HemECs. Under the inverted phase contrast microscope, before the treatment of TGF- $\beta 1$, the cells were irregular polygon with clear boundary and tight connection between cells. After 3 days of culture, the cells in the TGF- $\beta 1$ group changed significantly in morphology, elongated spindle type, fuzzy boundary, and large gap, from paving stone to shuttle shape.

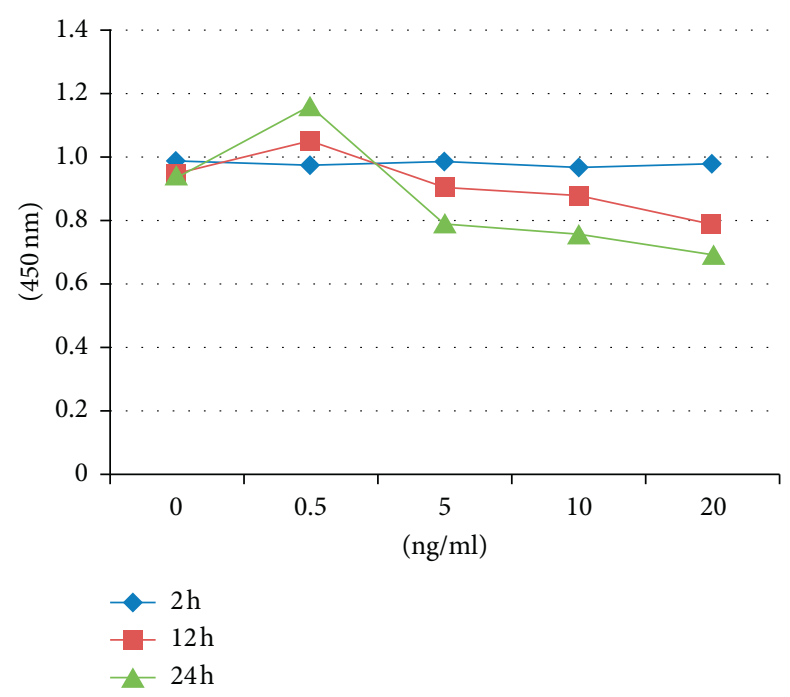

FIgURE 2: TGF- $\beta 1$ inhibits the proliferation of HemECs. The effect of transforming growth factor- $\beta 1$ (TGF- $\beta 1$ ) with different concentrations on proliferation of HemECs was detected by the CCK- 8 method.

\section{Discussion}

The transformation of epithelial cells into mesenchymal cells follows a common conservative procedure in physiology and pathology [12-15]. Endothelial cells, as a special subtype of epithelial cells, have recently been shown to undergo EndMT. EndMT is closely related to EMT, which defines the process of ECs losing endothelial cell characteristics and obtaining mesenchymal cell characteristics [16-20].

Previous efforts showed that EndMT can lead to the occurrence and development of cerebral cavernous malformation. Typical mesenchymal markers such as slug, DNA binding inhibitor 1 , and $\alpha$-SMA are highly expressed in regulating endothelial cells to form vascular malformation [21]. EndMT could increase the ability of cell migration, which is a key feature of activated fibroblasts. It is also found in the fibroblast-like cells derived from EndMT, which is consistent with our work that TGF- $\beta 1$ can enhance the ability of cell migration.

TGF- $\beta 1$, as one of the members of the TGF- $\beta$ family, has a significantly higher expression in the proliferative phase of hemangioma than in the regression phase 16 [22], and it is the most important EndMT inducer at present [23]. Li et al. confirmed that TGF $\beta /$ Smad is the key pathway to induce EndMT and EMT [24]. In the present work, we utilized TGF$\beta 1$ to intervene the endothelial cells of hemangioma and observed the changes in cell morphology and function.

TGF- $\beta 1$ could downregulate the expression of E-cadherin and cytokeratin and upregulate the expresssion of mesenchymal markers (vimentin, N-cadherin, and fibronectin) by binding to its receptor [25]. Endothelial differentiation and angiogenesis have been reported to be associated with EndMT. The molecular characterization of the Hem ENG + subgroup showed higher protein levels of VEZF1 and lower protein level of PROM1/SALL4, indicating the involvement of TGF-signaling in endothelial differentiation [25]. EndMT participates in the epicardial experience and produces resident stromal fibroblasts in the myocardium, as well as coronary smooth muscle cells and at least $20 \%$ of endothelial cells [26]. TGF- $\beta$ exerts a strong inhibitory effect on the growth of nontumor epithelial cells by promoting G1 arrest [26].

In the degenerative phase of hemangioma, the high level expression level of TGF-1 mRNA may be related to the increase in fibrous tissue [27] and vimentin is the main interstitial cell marker, which has a certain regulatory effect 

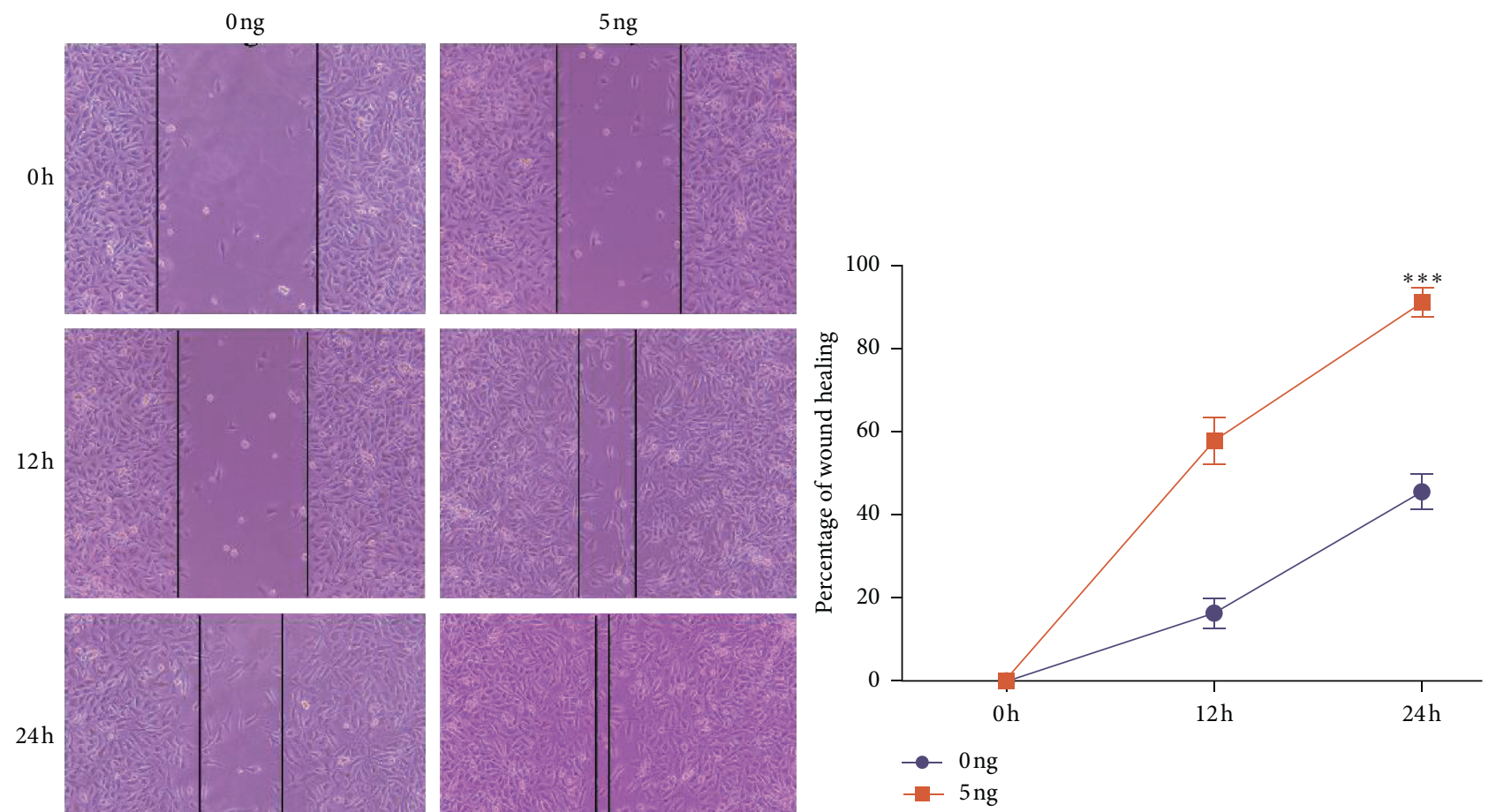

FIgure 3: TGF- $\beta 1$ promotes the migration of HemECs. The scratch distance of cells treated with $0 \mathrm{ng} / \mathrm{ml}$ and $5 \mathrm{ng} / \mathrm{ml}$ TGF- $\beta 1$ for 24 hours, in which group with $5 \mathrm{ng} / \mathrm{ml} \mathrm{TGF}-\beta 1$ was significantly smaller than that of the blank control group, ${ }^{* * *} P<0.001$ compared with control.

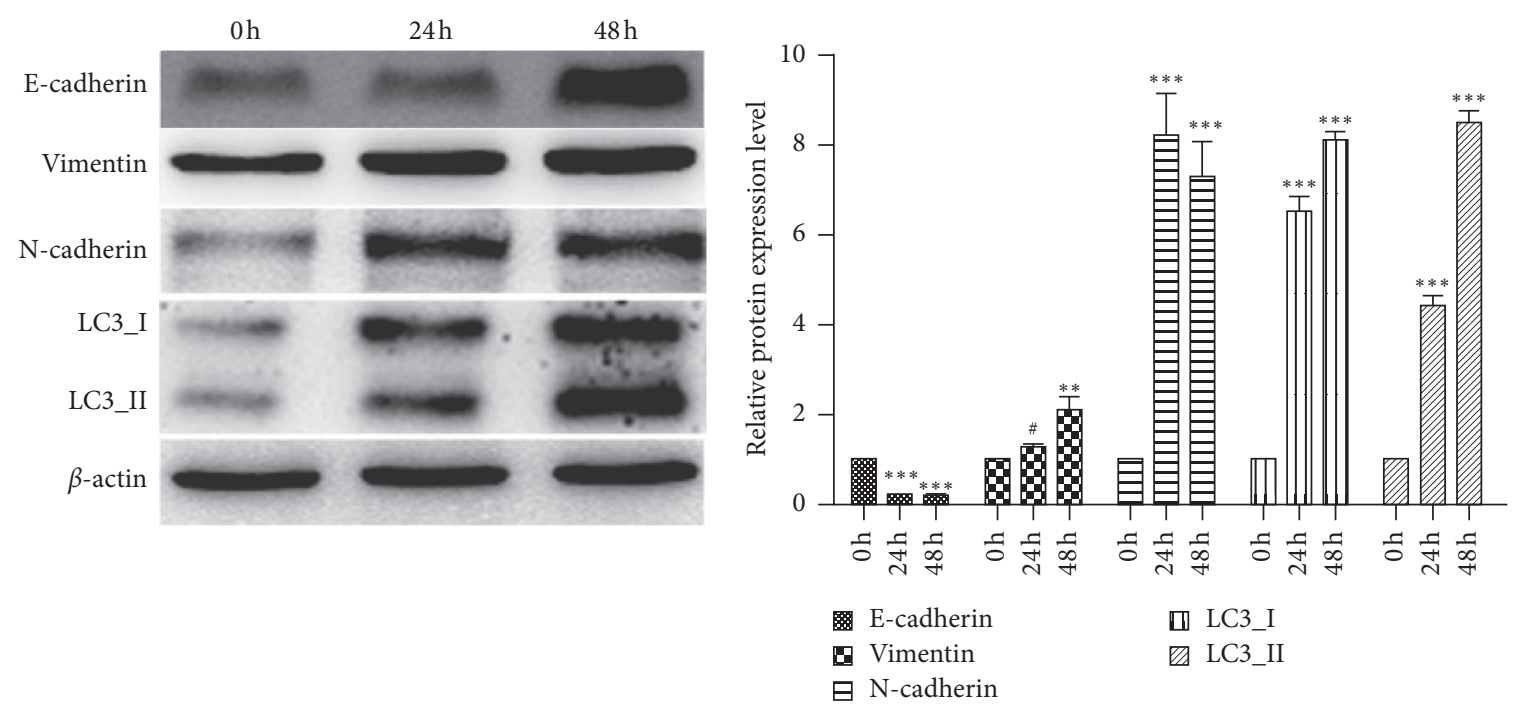

(a)

Figure 4: Continued. 


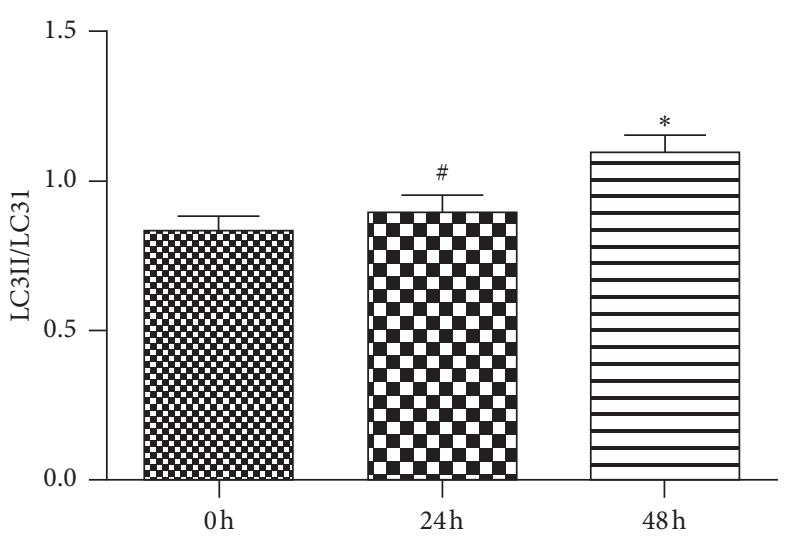

(b)
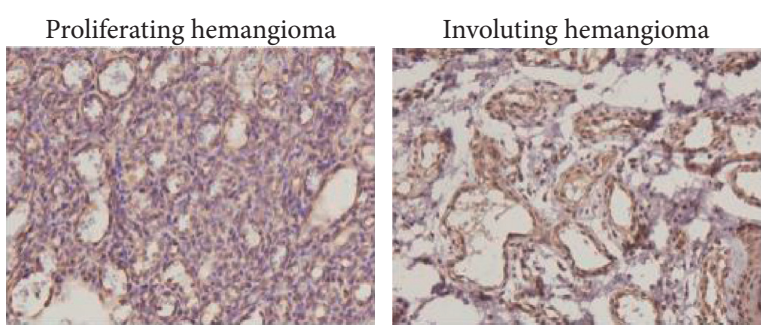

(c)

Figure 4: The effect of EndMT on the expression of endothelial and mesenchymal cell markers. (a) Protein expression of HemECs treated with TGF- $\beta 1$. (b) Western blot analysis of LC3 expression in HemECs. (c) Immunohistochemical staining for LC3 in infantile hemangioma tissues. ${ }^{\#} P<0.05 ;{ }^{*}{ }^{*} P<0.01 ;{ }^{*}{ }^{*}{ }^{*} P<0.001$.

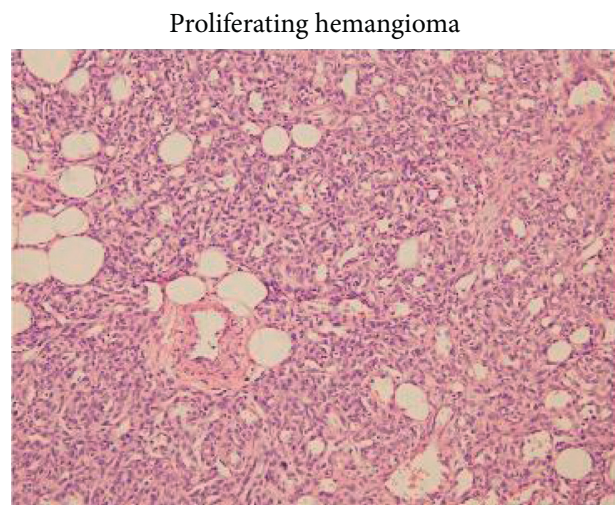

(a)

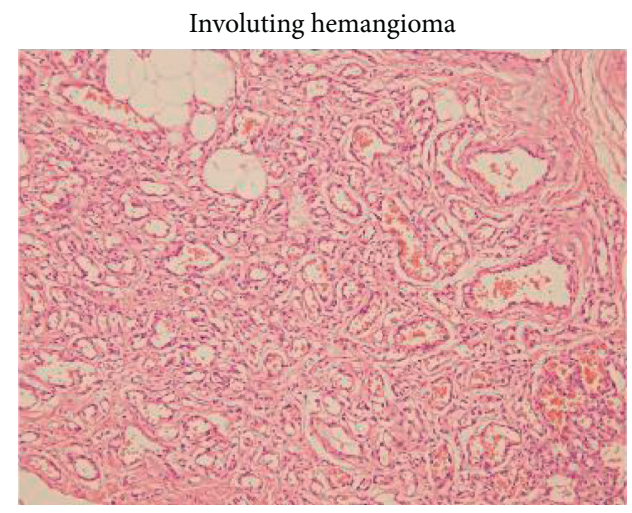

(b)

Figure 5: Structural changes of hemangioma tissue during EndMT. (a) Proliferative phase $(\mathrm{SP} \times 100)$. (b) Regressive phase $(\mathrm{SP} \times 100)$.
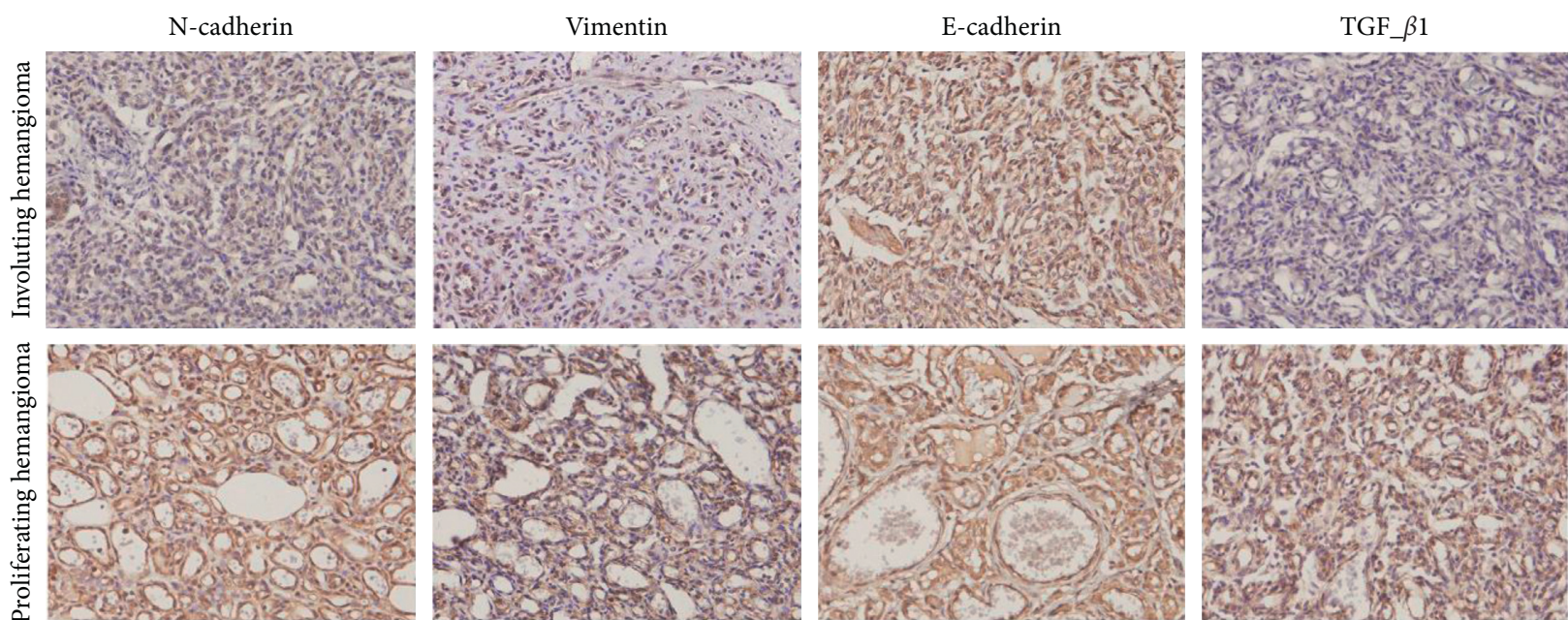

FIGURE 6: Expression of related genes in hemangioma during EndMT. Immunohistochemical staining of N-cadherin, vimentin, E-cadherin, and TGF- $\beta 1$ in IH tissues in different phases $(\mathrm{SP} \times 200)$. 
TABLE 1: Expression of TGF- $\beta 1$ in infantile hemangioma.

\begin{tabular}{lcccr}
\hline Clinical indicators & Case $(n)$ & TGF- $\beta 1$ & $-(\%)$ & $\chi^{2}$ \\
\hline Proliferation phase & 23 & $9(39.1)$ & $14(60.9)$ & 7.342 \\
Involution phase & 20 & $16(80.0)$ & $4(80.0)$ & $<0.01$ \\
\hline
\end{tabular}

TABLE 2: The correlation between E-cadherin and N-cadherin expression and clinicopathologic characteristics in IHC analysis.

\begin{tabular}{|c|c|c|c|c|c|c|c|}
\hline \multirow{2}{*}{ Clinical indicators } & \multirow{2}{*}{ Case $(n)$} & \multicolumn{3}{|c|}{ E-cadherin expression } & \multicolumn{3}{|c|}{$\mathrm{N}$-cadherin expression } \\
\hline & & $+(\%)$ & $-(\%)$ & $P^{*}$ & $+(\%)$ & $-(\%)$ & $P^{*}$ \\
\hline \multicolumn{8}{|l|}{ Diagnostic category } \\
\hline Proliferation phase & 23 & $22(95.7)$ & $1(4.3)$ & $<0.01$ & $6(26.1)$ & $17(73.9)$ & $<0.01$ \\
\hline Involution phase & 20 & $7(35)$ & $13(65)$ & & $20(100.00)$ & $0(0.00)$ & \\
\hline \multicolumn{8}{|l|}{ Gender } \\
\hline Male & 19 & $13(68.4)$ & $6(31.6)$ & 0.903 & $13(68.4)$ & $6(31.6)$ & 0.342 \\
\hline Female & 24 & $16(66.7)$ & $8(33.3)$ & & $13(54.2)$ & $11(45.8)$ & \\
\hline \multicolumn{8}{|l|}{ Age (years old) } \\
\hline$<1$ & 22 & $21(95.5)$ & $1(4.5)$ & $<0.01$ & $6(27.3)$ & $16(72.7)$ & $<0.01$ \\
\hline$\geq 1$ & 21 & $8(38.1)$ & $13(61.9)$ & & $20(95.2)$ & $1(4.8)$ & \\
\hline \multicolumn{8}{|l|}{$\overline{D e p t h}(\mathrm{~cm})$} \\
\hline$<0.5$ & 20 & $14(70.0)$ & $6(30.0)$ & 0.739 & $14(70.0)$ & $6(30.0)$ & 0.954 \\
\hline$\geq 0.5$ & 23 & $15(65.2)$ & $8(34.8)$ & & $12(52.2)$ & $11(47.8)$ & \\
\hline \multicolumn{8}{|l|}{ Size $\left(\mathrm{cm}^{2}\right)$} \\
\hline$<4$ & 21 & $15(71.4)$ & $6(28.6)$ & 0.586 & $12(57.1)$ & $9(42.9)$ & 0.663 \\
\hline$\geq 4$ & 22 & $14(63.6)$ & $8(36.4)$ & & $14(63.6)$ & $8(36.4)$ & \\
\hline \multicolumn{8}{|l|}{ Classification } \\
\hline Mixed type & 12 & $9(75)$ & $3(25)$ & 0.639 & $6(50.0)$ & $6(50.0)$ & 0.428 \\
\hline Superficial type & 22 & $15(68.2)$ & $7(31.8)$ & & $13(59.1)$ & $9(40.9)$ & \\
\hline Deep type & 9 & $5(55.6)$ & $4(44.4)$ & & $7(77.8)$ & $2(22.2)$ & \\
\hline
\end{tabular}

${ }^{*} P$ values are obtained from $\chi^{2}$ test.

on the EndMT process. Meanwhile, TGF- $\beta 1$, Wnt, mitogen activated protein kinase, epidermal growth factor receptor, phosphatidylinositol 3-kinase, and other signaling pathways can regulate the expression of vimentin and cause the phenotype change of EndMT. However, vimentin deletion can inhibit the process of EndMT, which is important for maintaining cell integrity.

Previous efforts suggested that in the process of TGF- $\beta 1$ induced EndMT, the cells changed from round lamellar growth to long fusiform, and the connections between cells were weakened and dispersed [28]. In our work, we confirmed that the morphology of HemECs induced by TGF- $\beta 1$ changed significantly. The elongated cells showed a fusiform change, the boundary became fuzzy, the gap became larger, and the transition from paver-like to fusiform was consistent with the morphological change of EndMT. In addition, we also detected the expression of related proteins E-cadherin, vimentin, and $\mathrm{N}$-cadherin via Western blot, which further confirmed that TGF- $\beta 1$ successfully induced the EndMT model.

Nieto et al. proposed that there was an intermediate stage spectrum between epithelial cells and stromal cells when epithelial cells transformed into EMT. In the course of EMT, the loss of epithelial marker expression may lead to the acquisition of stromal markers. When EMT pressure persists, they either complete the transformation to mesenchymal cells or reverse the epithelial phenotype when the pressure disappears [29]. The above results suggest that endothelial cells, a special subtype of epithelial cells, can subside by themselves in some hemangiomas, but not in all hemangiomas, maybe affected by EndMT pressure.

During the course of EndMT, the expression of these endothelial markers decreased significantly although some minimal levels of expression were generally maintained. Interstitial specific gene expression includes vimentin, smooth muscle actin (SMA), and N-cadherin [30].

It has not been clear for a long time whether this angiogenic switch is reflected by metabolic switch. LC3 is the main Atg8 homolog detected in mammalian cells and is a typical autophagic small body marker. LC3 protein exists in cells in the form of LC3-I and LC3-II. During the formation of autophagy, LC3-II gradually accumulates on the autophagy membrane and increases gradually. In this work, we confirmed that the protein levels of LC3 and LC3-II/LC3-I both increased markedly after TGF- $\beta 1$ treatment. It is suggested that the endothelial cells of hemangioma may increase autophagy function through EndMT and reduce cell metabolism and maintain normal cell life activity.

In conclusion, TGF- $\beta 1$ could induce HemECs to lose cobblestone-like morphology and present fusiform or fibroblast-like appearance, inhibit proliferation activity, promote endothelial cell migration, reduce endothelial cell 
markers, increase the expression of interstitial markers, and induce autophagy. In addition, the autophagy level of EndMT was higher in the degenerative stage than in the proliferative stage, which indicated that EndMT might promote the degradation of hemangioma. LC3B was lower in the proliferative stage than that in the regressive stage in immunohistochemistry. Our work confirmed that hemangioma is closely related to EndMT, which may provide a new insight for the treatment of hemangioma.

\section{Materials and Methods}

4.1. Patients and Specimens. A total of 43 cases of hemangioma diagnosed clinically and pathologically were collected from Xuzhou Central Hospital from January 2018 to October 2019. All patients were treated by operation without any other treatment (see Table 1 for specific clinical data). The average age of the patients was $(2.07 \pm 1.92)$ years old, 19 male and 24 female. The lesions were distributed in the face, neck, trunk, and limbs, including 23 cases in proliferative stage and 20 cases in regressive stage. The work was approved by the ethics committee of Xuzhou Central Hospital, and all patients agreed and signed the informed consent.

4.2. Cell Isolation and Culture. TGF- $\beta 1$ was obtained from PeproTech (Suzhou, China), DMEM with high glucose culture medium was obtained from Keygene biology (Nanjing, China), and fetal bovine serum (FBS) was obtained from GIBCO (Grand Island, NY, USA). A sample of hemangioma of infant was cut to the size of $1-2 \mathrm{~cm}^{3}$. After 3 times of washing with PBS solution preheated at $37^{\circ} \mathrm{C}$, the tissue block was put into the centrifuge tube. Then, a proper amount of preheated trypsin solution was added, it was shaken in a $37^{\circ} \mathrm{C}$ water bath, and digestion was stopped after the tissue structure becomes loose. The tissue was put into the culture dish, and it was washed with PBS solution for three times. After discarding the cleaning solution, appropriate amount of culture medium was added to submerge the tissue, the visible fat tissue was cut off, the tumor tissue was cut to the size of $1-2 \mathrm{~mm}^{3}$, then it was inoculated to the bottom of the culture bottle, and it was put into a $37^{\circ} \mathrm{C}$ incubator with $5 \% \mathrm{CO}_{2}$. After 8 hours, $1 \mathrm{ml}$ high glucose DMEM containing 10\% FBS was added for culture, and the medium was changed every day. After the fusiform or irregular cells occupy more than $75 \%$ of the culture flask, the tissue block was removed and $4 \mathrm{ml}$ of complete culture medium was added to the culture flask. After the number of cells accounted for more than $80 \%$ of the bottom area of the bottle, they were subcultured. Finally, the expression of endothelial cell marker CD31 in HemECs was detected by immunohistochemistry.

4.3. CCK-8 Assay. Total $2 * 10^{3}$ HemECs per well were inoculated into 96 -well plate, cultured in $37^{\circ} \mathrm{C}$ with $5 \% \mathrm{CO}_{2}$ for 24 hours, then treated with TGF- $\beta 1(0,0.5 \mathrm{ng} / \mathrm{ml}, 5 \mathrm{ng} / \mathrm{ml}$, $10 \mathrm{ng} / \mathrm{ml}$, and $20 \mathrm{ng} / \mathrm{ml}$ ), each group with 5 multiple wells, and set up blank control. When cultured for 2, 12, and 24 hours, respectively, $10 \mathrm{uL}$ of CCK-8 (Dojindo Chemical
Laboratory, Kumamoto, Japan) reagent was added to each hole and incubated in a constant temperature incubator at $37^{\circ} \mathrm{C}$ for 2 hours. The OD value of each hole was measured at the wavelength of $450 \mathrm{~nm}$ by enzyme-linked immunosorbent assay.

4.4. Scratch Analysis. The cells were inoculated into 6-well plates with $5 \times 10^{5}$ cells per well. After fusion, the cells were scratched horizontally and vertically with a $100 \mu \mathrm{l}$ micropipette, and the width was controlled at about $500 \mu \mathrm{M}$. PBS solution was washed three times along the side wall and cultured in a $37^{\circ} \mathrm{C}$ with $5 \% \mathrm{CO}_{2}$ incubator. After 0,12 , and 24 hours, pictures were taken under Zeiss microscope.

4.5. Western Blot Analysis. HemECs treated with TGF- $\beta 1$ were collected, and the total protein of each group was extracted by cell lysate buffer. The protein concentration was measured by BCA (BCA, Beijing Biotechnology, China) methods. Then, the proteins $(100 \mathrm{~V}$ constant pressure) were separated and transferred to PVDF membrane $(300 \mathrm{~mA}$ constant current) at $4^{\circ} \mathrm{C}$ using $6 \%$ or $12 \%$ SDS-PAGE. The membrane was incubated in PBS with 5\% skimmed milk at room temperature for 1 hour and then reacted overnight at $4^{\circ} \mathrm{C}$ in an anti-E-cadherin (3195, $1: 1000$, CST, USA), vimentin (5741, $1: 1000$, CST, USA), N-cadherin (13116, 1 : 1000, CST, USA), LC3B (3868, $1: 1000$, CST, USA), and $\beta$-actin monoclonal antibody $(1: 1000$, protein, China). After it was washed 3 times with TBST, secondary antibody dilutions were added (AS014, 1:10000, Abclonal) at room temperature for $2 \mathrm{~h}$. Last, semiquantification of protein bands was carried out by using Tanon TM High-sig ECL Western Blotting Substrate (Tanon, Shanghai, China).

4.6. Immunohistochemical Detection. All specimens were fixed with $10 \%$ neutral formaldehyde and embedded in paraffin. The tissue wax samples were sectioned continuously for $4 \mu \mathrm{m}$, baked, dewaxed, inactivated endogenous peroxidase, and heat repaired with high pressure antigen. $5 \%$ BSA solution and primary antibody solution cadherin (1: 400 dilution; CST, USA), vimentin (1:200 dilution; CST, USA), N-cadherin (1:200 dilution; CST, USA), and LC3B (1:1000 dilution CST, USA) were added. Then, we added biotinylated IgG, DAB (Zhongshan Biotechnology Company, Beijing, China), for color development, stained with hematoxylin and differentiated with hydrochloric acid for a few seconds, washed with distilled water for $1 \mathrm{~min}$ and treated with lithium carbonate for $1 \mathrm{~min}$, and washed with distilled water for $30 \mathrm{~s}-1 \mathrm{~min}$. Alcohol is dehydrated step by step, xylene was transparent, and neutral gum was added with cover glass. Finally, the sections were photographed with the Olympus bx-51 optical microscope.

The results of immunohistochemistry were analyzed by two experienced pathologists. At high magnification $(\times 400)$ of each section, 10 fields of vision were randomly selected, and 100 cells were counted for each field of vision. The consensus was reached, the percentage of positive cells was calculated, and then the average value was calculated. 
Judgment criteria are as follows: (1) the degree of cell staining (contrast of staining depth and background color): 0 (no staining), 1 (light yellow), and 2 (brown yellow), and 3 (brown brown brown) and (2) the percentage of positive cells: 0 (no positive cell expression), 1 (positive cell $\leq 10 \%$ ), 2 (positive cell 11\%-50\%), 3 (positive cell 51\%-75\%), and 4 (positive cell $>75 \%$ ). Finally, according to the product of the above two scores, $\leq 3$ points are negative expression and $>3$ points are positive expression.

4.7. Statistical Analysis. Statistical analysis was performed using SPSS 22.0. Data were presented as mean \pm standard deviation (SD). The difference between means was analyzed with Student's $t$-test. $P<0.05, P<0.01$, or $P<0.001$ was considered to indicate a statistically significant.

\section{Data Availability}

The data utilized to support the work are included within the manuscript.

\section{Conflicts of Interest}

The authors declare that they have no conflicts of interest.

\section{Authors' Contributions}

Xin Zhong edited the manuscript of this work. Qi-Hong Qian designed the experiment of this work. Gang Zheng collected the data of this work. Min Ding and Jing-Bing Li contributed significantly to analysis and manuscript preparation.

\section{References}

[1] I. Adini, K. Ghosh, A. Adini et al., "Melanocyte-secreted fibromodulin promotes an angiogenic microenvironment," Journal of Clinical Investigation, vol. 124, no. 1, pp. 425-436, 2014.

[2] E. Cano, R. Carmona, A. Ruiz-Villalba et al., "Extracardiac septum transversum/proepicardial endothelial cells pattern embryonic coronary arterio-venous connections," in Proceedings of the National Academy of Sciences, vol. 113, no. 3, pp. 656-661, 2016.

[3] J. Chen, J. Jia, L. Ma et al., "Nur77 deficiency exacerbates cardiac fibrosis after myocardial infarction by promoting endothelial-to-mesenchymal transition," Journal of Cellular Physiology, vol. 236, no. 1, pp. 495-506, 2020.

[4] S. Chen, K. Zhuang, K. Sun et al., "Itraconazole induces regression of infantile hemangioma via downregulation of the platelet-derived growth factor-D/PI3K/Akt/mTOR pathway," Journal of Investigative Dermatology, vol. 139, no. 7 , pp. 1574-1582, 2019.

[5] B. C. Cooley, J. Nevado, J. Mellad et al., "TGF- signaling mediates endothelial-to-mesenchymal transition (EndMT) during vein graft remodeling," Science Translational Medicine, vol. 6, no. 227, p. 227ra34, 2014.

[6] V. Das, S. Bhattacharya, C. Chikkaputtaiah, S. Hazra, and M. Pal, "The basics of epithelial-mesenchymal transition (EMT): a study from a structure, dynamics, and functional perspective," Journal of Cellular Physiology, vol. 234, no. 9, pp. 14535-14555, 2019.

[7] H. A. Dbouk, L. M. Weil, G. K. S. Perera et al., "Actions of the protein kinase WNK1 on endothelial cells are differentially mediated by its substrate kinases OSR1 and SPAK," Proceedings of the National Academy of Sciences, vol. 111, no. 45, pp. 15999-16004, 2014.

[8] V. Ellenrieder, S. F. Hendler, W. Boeck et al., "Transforming growth factor betal treatment leads to an epithelial-mesenchymal transdifferentiation of pancreatic cancer cells requiring extracellular signal-regulated kinase 2 activation," Cancer Research, vol. 61, no. 10, pp. 4222-4228, 2001.

[9] S. M. Evrard, L. Lecce, K. C. Michelis et al., "Endothelial to mesenchymal transition is common in atherosclerotic lesions and is associated with plaque instability," Nature Communications, vol. 7, no. 1, p. 11853, 2016.

[10] Y. Fu, Z. G. Yang, and L. Y. Zhao, "Angiogenesis characteristics of infantile hemangioma and feasibility observation of transplantation model of human hemangioma on mice," European Review for Medical and Pharmacological Sciences, vol. 21, no. 6, pp. 1276-1280, 2017.

[11] S. Gupta and A. Maitra, "EMT: matter of life or death?" Cell vol. 164, no. 5, pp. 840-842, 2016.

[12] S. Harbi, R. Wang, M. Gregory et al., "Infantile hemangioma originates from A dysregulated but not fully transformed multipotent stem cell," Scientific Reports, vol. 6, no. 1, p. $35811,2016$.

[13] W. Bao, D.-S. Huang, and Y.-H. Chen, "MSIT: malonylation sites identification tree," Current Bioinformatics, vol. 15, no. 1, pp. 59-67, 2020.

[14] J. C. Kovacic, S. Dimmeler, R. P. Harvey et al., "Endothelial to mesenchymal transition in cardiovascular disease," Journal of the American College of Cardiology, vol. 73, no. 2, pp. 190-209, 2019.

[15] W. Bao, B. Yang, Z. Li, and Y. Zhou, "LAIPT: lysine acetylation site identification with polynomial tree," International Journal of Molecular Sciences, vol. 20, no. 1, p. 113, 2018.

[16] F. Laurent, A. Girdziusaite, J. Gamart et al., "HAND2 target gene regulatory networks control atrioventricular canal and cardiac valve development," Cell Reports, vol. 19, no. 8, pp. 1602-1613, 2017.

[17] J. Li, S. Shi, S. P. Srivastava et al., "FGFR1 is critical for the anti-endothelial mesenchymal transition effect of $\mathrm{N}$-acetyl-seryl-aspartyl-lysyl-proline via induction of the MAP4K4 pathway," Cell Death \& Disease, vol. 8, no. 8, p. e2965, 2017.

[18] Y. Li, K. O. Lui, and B. Zhou, "Reassessing endothelial-tomesenchymal transition in cardiovascular diseases," Nature Reviews Cardiology, vol. 15, no. 8, pp. 445-456, 2018.

[19] W. Bao, B. Yang, D. Li, Z. Li, Y. Zhou, and R. Bao, "CMSENN: computational modification sites with ensemble neural network," Chemometrics and Intelligent Laboratory Systems, vol. 185, pp. 65-72, 2019.

[20] W. Bao, B. Yang, D.-S. Huang et al., "IMKPse: identification of protein malonylation sites by the key features into general PseAAC," IEEE Access, vol. 7, pp. 54073-54083, 2019.

[21] W. Bao, C.-A. Yuan, Y. Zhang et al., "Mutli-features prediction of protein translational modification sites," IEEE/ ACM Transactions on Computational Biology and Bioinformatics, vol. 15, no. 5, pp. 1453-1460, 2018.

[22] M. A. Nieto, R. Y.-J. Huang, R. A. Jackson, and J. P. Thiery, "EMT: 2016," Cell, vol. 166, no. 1, pp. 21-45, 2016.

[23] E. Pardali, G. Sanchez-Duffhues, M. Gomez-Puerto, and P. Ten Dijke, "TGF- $\beta$-Induced endothelial-mesenchymal 
transition in fibrotic diseases," International Journal of Molecular Sciences, vol. 18, no. 10, p. 2157, 2017.

[24] J. A. Weston and J. P. Thiery, "Pentimento: neural crest and the origin of mesectoderm," Developmental Biology, vol. 401, no. 1, pp. 37-61, 2015.

[25] W. Bao, D. Wang, and Y. Chen, "Classification of protein structure classes on flexible neutral tree," IEEE/ACM Transactions on Computational Biology and Bioinformatics, vol. 14, no. 5, pp. 1122-1133, 2017.

[26] Q. Xiao, H. Liu, H.-S. Wang et al., "Histone deacetylase inhibitors promote epithelial-mesenchymal transition in $\mathrm{He}$ patocellular Carcinoma via AMPK-FOXO1-ULK1 signaling axis-mediated autophagy," Theranostics, vol. 10, no. 22, pp. 10245-10261, 2020.

[27] T. Yamashita, M. Jinnin, K. Makino et al., "Serum cytokine profiles are altered in patients with progressive infantile hemangioma," Bioscience Trends, vol. 12, no. 4, pp. 438-441, 2018.

[28] W. L. Yuan, X. J. Qin, and X. K. Wang, "Expression and correlation of mast cell, Clusterin/apoJ and transforming growth factor-beta in the different stages of human dermal hemangioma," Hua Xi Kou Qiang Yi Xue Za Zhi = Huaxi Kouqiang Yixue Zazhi = West China Journal of Stomatology, vol. 27, no. 4, pp. 361-365, 2009.

[29] W. Bao, Z. Huang, C. A. Yuan et al., "Pupylation sites prediction with ensemble classification model," International Journal of Data Mining and Bioinformatics, vol. 18, no. 2, pp. 91-104, 2017.

[30] P. X. Zhao, Y. M. Adzavon, J. M. Ma et al., "IgG4 and IgE copositive group found in idiopathic orbital inflammatory disease," International Journal of Ophthalmology, vol. 11, no. 1, pp. 36-42, 2018. 